\title{
Sex prediction by metric and non-metric analysis of the hard palate and the pyriform aperture
}

\author{
N. Alves ${ }^{1,2,3}$, N.F. Deana ${ }^{4}$, F. Ceballos³, P. Hernández³, J. González ${ }^{3}$ \\ ${ }^{1}$ Research Centre of Applied Morphology, Universidad de La Frontera, Temuco, Chile \\ ${ }^{2}$ Centre of Excellence in Morphological and Surgical Sciences, Universidad de La Frontera, Temuco, Chile \\ ${ }^{3}$ Dental School, Universidad de La Frontera, Temuco, Chile \\ ${ }^{4}$ Master Programme in Dentistry, Faculty of Dentistry, Universidad de La Frontera, Temuco, Chile
}

[Received: 4 May 2018; Accepted: 8 June 2018]

Background: Analysis of the bones and bone fragments of the cranium may be a useful tool for sex diagnosis in the identification of human remains which have been exposed to adverse conditions. The object of the present study was to evaluate sex prediction through metric and non-metric analysis of the hard palate (HP) and the pyriform aperture (PA), using macerated skulls of adult individuals. Materials and methods: We analysed 312 dry skulls of adult individuals of both sexes, studying the metric and non-metric characteristics of the HP and PA. The accuracy, sensitivity, specificity and positive and negative predictive values were evaluated. A binary logistic regression and a linear regression were performed. The receiver operating characteristic curve was constructed to analyse the performance of sex diagnosis. Measurements of the HP and the PA were analysed by ANOVA and Tukey's test. The SPSS v. 20.0 software was used, with a significance threshold of $5 \%$.

Results: The shape of the PA presented $61.9 \%$ accuracy, $54.4 \%$ sensitivity and $65.7 \%$ specificity. The shape of the HP presented $51.5 \%$ accuracy, $65.6 \%$ sensitivity and $44.7 \%$ specificity. Only the height of the PA functioned as a good predictor of sex.

Conclusions: The height of the PA produced good diagnostic performance (area under curve $=0.764$ ). The height of the PA was the most reliable indicator for sex prediction, and could be used by forensic scientists to identify sex. (Folia Morphol 2019; 78, 1: 137-144)

Key words: pyriform aperture, hard palate, sex prediction

\section{INTRODUCTION}

Forensic anthropology is a multidisciplinary field dealing with a variety of matters, including individual identification [8]. The need to identify human remains arises both naturally and as a result of man-made disasters, when corpses are difficult to identify due to their advanced decomposition, dismemberment, mutilation or for any other reason [21] Determining sex is a fundamental part of creating a reliable biological profile when examining skeletal remains, and is also vital in estimating age and descent $[29,30]$.

The cranium is considered the second most reliable bone structure for determining the sex of an adult individual [5]. Distortion of the soft tissues and bone fragmentation due to heat may make it difficult to determine sex [12]. The characteristics of the pyriform aperture (PA) offer a classic sex indicator [7]. The PA communicates the nasal cavity with the exterior; its superior limit is determined by the nasal bones and its 


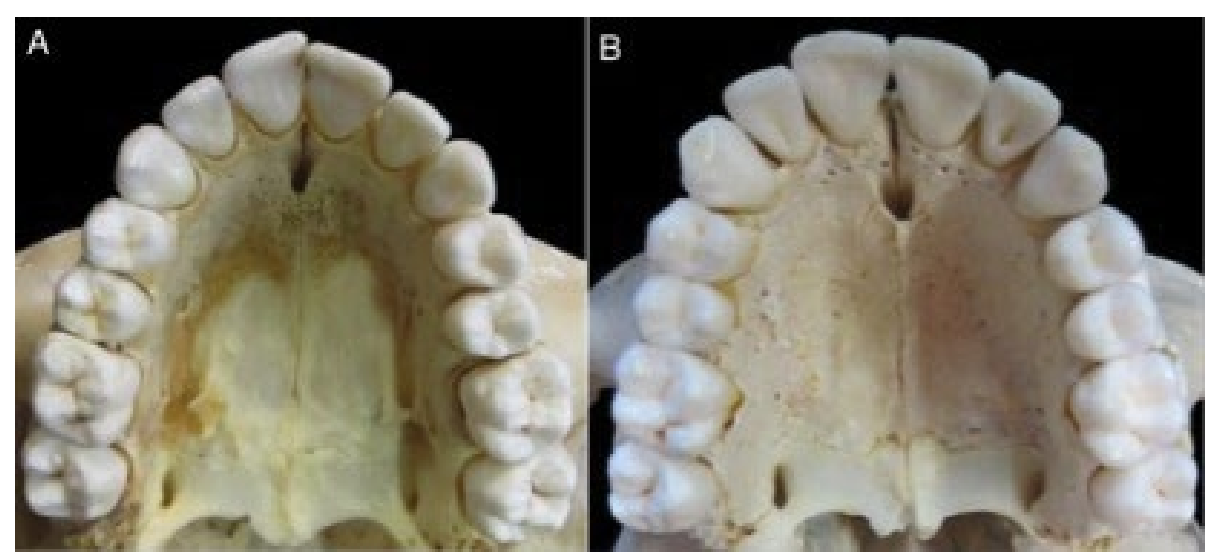

Figure 1. Non-metric characteristics of the hard palate. A. Female; B. Male.

lateral and inferior limits by the maxilla [2]. Anthropological studies suggest that the shape of the PA is influenced by congenital anatomical variations [14] and climatic factors [34], adapting to the geographical conditions in which the population lives [16]. The osseous anatomical structures located in the base region of the cranium are very strong, maintaining their vertical dimensions even when subjected to high temperatures; they are therefore ideal for sex determination [13]. The hard palate (HP) is the osseous anterior part of the palate [2], formed by the union of the palatine processes of the maxilla and horizontal plates of the palatine bones, united by a cruciform suture [28]. The HP is highly resistant, and is located in isolation in the base of the cranium. It is therefore often available for forensic analysis, even when other superficial skeletal components are damaged [13].

Both metric and non-metric (analysis of characteristics) methods are often used for determining the sex of human remains. The simplest methods are used initially, but when the remains are decomposed or incomplete and present insufficient information for diagnosis, more expensive and sophisticated complementary methods are required [21]. The object of the present study was to evaluate sex prediction through metric and non-metric analysis of the hard palate and the pyriform aperture, using macerated skulls of adult individuals.

\section{MATERIALS AND METHODS}

\section{Samples}

We analysed 312 macerated skulls of adult individuals, of both sexes, viz. 104 women and 208 men, aged between 18 and 100 years. They belonged to the Department of Morphology and Genetics, UNIFESP (Brazil). Skulls for which there was no information on sex, damaged skulls and those which presented any kind of pathology were excluded from this study. The investigators were calibrated in advance for both the metric and non-metric analyses. The investigators were blinded for the sex diagnosis, being unaware of the real identification of the skulls when they made their determination.

\section{Analysis of non-metric characteristics}

The non-metric characteristics of the HP and PA were analysed by a single investigator after prior calibration. The palate was classified as male when it was U-shaped and female when it was V-shaped (Fig. 1) [32]. The pyriform aperture was classified as male when it was high with fine margins and female when it was lower with rounded margins (Fig. 2).

\section{Analysis of metric characteristics}

The PA and HP were measured with a digital calliper.

Five measurements were taken of the HP for metric analysis: 1 . Distance between the right and left greater palatine foramina (RGPF-LGPF); 2 . Distance between the incisive fossa (IF) and the mid-point between the greater palatine foramina (GPF) (IF-GPF); 3. Maxillary alveolar length, i.e. the distance from the prosthion to the alveolon; 4. Maxillary alveolar amplitude, i.e. the greatest width of the alveolar process of the maxilla, measured in the vestibular faces of the maxilla at the level of the second molars; 5 . Palate size, i.e. the maxillary alveolar length $\times$ maxillary alveolar amplitude / 100 [22].

Three measurements were taken of the PA for metric analysis: 1 . Height of the pyriform aperture $(\mathrm{H})$, measured from the inferior edge of the internasal suture to the base of the anterior nasal spine; 


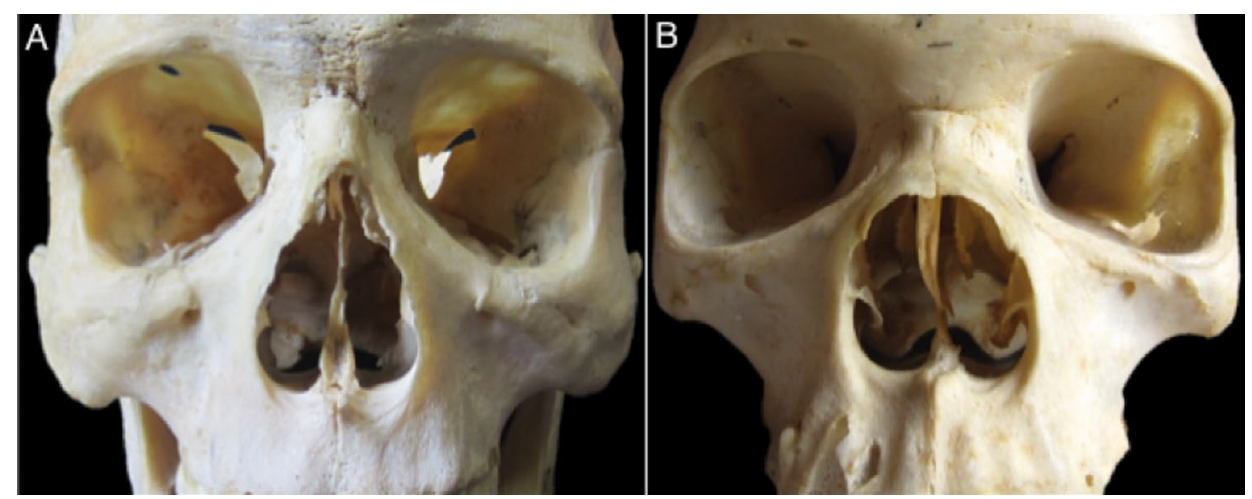

Figure 2. Non-metric characteristics of the pyriform aperture. A. Male; B. Female.

Table 1. Percentage of skulls diagnosed as male and female found to belong to men and women, respectively

\begin{tabular}{|c|c|c|c|c|c|c|c|c|}
\hline \multirow[t]{2}{*}{ Description } & \multirow[t]{2}{*}{ Sex } & \multicolumn{2}{|c|}{ Woman } & \multicolumn{2}{|c|}{ Man } & \multicolumn{2}{|c|}{ Total } & \multirow[t]{2}{*}{$\mathbf{P}$} \\
\hline & & $\mathbf{N}$ & $\%$ & $\mathbf{N}$ & $\%$ & $\mathbf{N}$ & $\%$ & \\
\hline \multirow[t]{2}{*}{ Pyriform aperture } & Female & 49 & $54 \%$ & 60 & $34 \%$ & 109 & $41 \%$ & 0.002 \\
\hline & Male & 41 & $46 \%$ & 115 & $66 \%$ & 156 & $59 \%$ & \\
\hline \multirow[t]{2}{*}{ Hard palate } & Female & 63 & $66 \%$ & 110 & $55 \%$ & 173 & $59 \%$ & 0.091 \\
\hline & Male & 33 & $34 \%$ & 89 & $45 \%$ & 122 & $41 \%$ & \\
\hline
\end{tabular}

$\mathrm{N}$ - number of skulls analysed; \% - percentage

2. Superior width (SW), i.e. the distance between the extremities of the inferior margin of the nasal bones; 3. Inferior width (IW), i.e. the greatest distance between the lateral margins of the pyriform aperture.

\section{Statistical analysis}

Descriptive analysis was by means with standard deviation. The measurements were compared by ANOVA. Chi-squared test was used for the qualitative variables. A linear regression model was used to verify whether the measurements taken for the PA and HP are accurate sex predictors. A binary logistic regression was used to verify whether the morphological characteristics of the PA and HP are sex predictors. The sensitivity, specificity, accuracy and positive and negative predictive values for the non-metric variables were also analysed, and a receiver operating characteristic curve was constructed establishing a cut-off point for each measurement. The SPSS v.20.0 software was used, with a significance threshold of $5 \%$.

\section{RESULTS}

\section{Non-metric characteristics}

By palate shape, $66 \%$ of the women's crania were classified as female, and $45 \%$ of the men's crania as male, with no statistical significance (Table 1). The
Table 2. Sensitivity and specificity for analysis of the morphological characteristics of the pyriform aperture (PA) and the hard palate (HP)

\begin{tabular}{lcc}
\hline & PA & HP \\
\hline Accuracy & $61.9 \%$ & $51.5 \%$ \\
Sensitivity & $54.4 \%$ & $65.6 \%$ \\
Specificity & $65.7 \%$ & $44.7 \%$ \\
PPV & $45.0 \%$ & $36.4 \%$ \\
NPV & $73.7 \%$ & $73.0 \%$ \\
\hline
\end{tabular}

PPV — positive predictive value; NPV — negative predictive value

sensitivity and specificity values presented a good balance, but were not very high; the negative predictive value (NPV) presented a good percentage while the positive predictive value (PPV) was low (Table 2). The binary logistic regression model for the HP was not significant $(p=0.070)$.

By PA, $54 \%$ of the women's crania were classified as female, and $66 \%$ of the men's crania as male, with significant results (Table 1). There was a good balance between sensitivity and specificity, but with low values; the NPV presented a good percentage while the PPV was low (Table 2). The binary logistic regression model for the PA as a sex predictor was 
Table 3. Mean values ( $\mathrm{mm}$ ), standard deviation (SD) and $\mathrm{p}$ value found for the hard palate by sex

\begin{tabular}{lccccc}
\hline Hard palate & Sex & N & Mean & SD & P \\
\hline RGPF-LGPF & 198 & Male & 30.67 & 2.94 & 0.339 \\
IF-GPF & 96 & Female & 30.31 & 2.76 & \\
& 199 & Male & 28.09 & 3.39 & 0.004 \\
MA length & 96 & Female & 26.78 & 3.43 & \\
& 200 & Male & 56.67 & 4.80 & 0.003 \\
MA amplitude & 96 & Female & 55.17 & 4.43 & \\
& 172 & Male & 62.41 & 5.21 & 0.007 \\
Palate size & 82 & Female & 60.31 & 4.24 & \\
& 176 & Male & 35.29 & 7.28 & 0.030 \\
& 84 & Female & 33.73 & 6.78 & \\
\hline
\end{tabular}

RGPF-LGPF — distance between the left and right greater palatine foramina; IF-GPF distance between the incisive fossa (IF) and the mid-point between the greater palatine foramina (GPF); MA — maxillary alveolar; $\mathrm{N}$ - number of skulls analysed

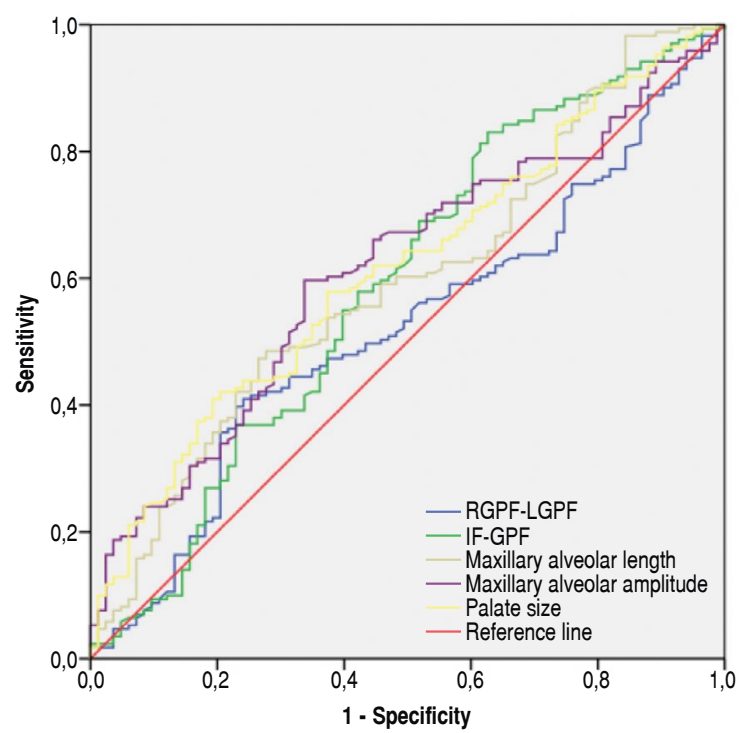

Figure 3. Receiver operating characteristic curve for the measurements taken in the hard palate; RGPF-LGPF — distance between the left and right greater palatine foramina; IF-GPF — distance between the incisive fossa (IF) and the mid-point between the greater palatine foramina. significant, but the model was weak $\left[\mathrm{X}^{2}(1)=9.751\right.$; $\mathrm{p}=0.002, \mathrm{R}^{2}$ Nagelkerke 0.048 ]. The shape of the $\mathrm{PA}$ was a significant predictor (odds ratio $=0.442$; $95 \%$ confidence interval $=0.264-0.740$ ).

\section{Metric characteristics}

In the HP, statistical differences were found for all the distances except RGPF-LGPF. The mean values observed for men were greater than for women in all the measurements. The low coefficient of variation showed homogeneity in the results (Table 3 ). The RGPF-LGPF, maxillary alveolar length and palate size presented low sensitivity and good specificity. The IF-GPF distance presented high sensitivity and moderate accuracy; and the maxillary alveolar amplitude presented good sensitivity/specificity balance and moderate accuracy (Table 4). We observed that RGPF-LGPF presented low diagnostic performance, with area under the curve (AUC) very close to 0.5 (AUC $=0.537$ ). The other distances measured for the HP presented regular diagnostic performance, with AUC of 0.604-0.617 (Fig. 3, Table 4). In the linear regression model we observed that none of the measurements for the hard palate presented good sex prediction (Table 5).

The mean values for the height of the PA found for men were significantly greater than those found for women. The superior and inferior widths presented higher mean values for males than for females, but with no statistical significance (Table 6). Only the height of the PA presented a good capacity for sex prediction; it also presented good balance between sensitivity and specificity and good accuracy (Table 7). According to the AUC analysis, the height of the PA presented good diagnostic performance (AUC $=0.764$ ), while the other distances for the PA (superior and inferior widths) presented low diagnostic performance with the AUC very close to 0.5 (Fig. 4, Table 7).

Table 4. Analysis of the receiver operating characteristic curve for the hard palate

\begin{tabular}{lccccccc}
\hline Hard palate & AUC & CP & Accuracy & Sensitivity & Specificity & PPV & NPV \\
\hline RGPF-LGPF & 0.537 & 31,460 & $53.7 \%$ & $43.4 \%$ & $75.0 \%$ & $78.2 \%$ & $39.1 \%$ \\
IF-GPF & 0.604 & 25,550 & $67.0 \%$ & $80.3 \%$ & $39.6 \%$ & $73.3 \%$ & $49.4 \%$ \\
MA length & 0.607 & 57,230 & $55.4 \%$ & $44.9 \%$ & $77.1 \%$ & $80.2 \%$ & $40.4 \%$ \\
MA amplitude & 0.615 & 61,300 & $62.0 \%$ & $59.9 \%$ & $66.3 \%$ & $78.6 \%$ & $44.4 \%$ \\
Palate size & 0.617 & 36,760 & $53.6 \%$ & $41.2 \%$ & $80.8 \%$ & $82.4 \%$ & $38.7 \%$ \\
\hline
\end{tabular}

RGPF-LGPF — distance between the left and right greater palatine foramina; IF-GPF — distance between the incisive fossa (IF) and the mid-point between the greater palatine foramina (GPF); MA — maxillary alveolar; AUC — area under curve; CP — cut-off point; PPV — positive predictive value; NPV — negative predictive value 
Table 5. Coefficients of the logistic regression model

\begin{tabular}{lcc}
\hline & Coefficient & P \\
\hline Constant & 2.562 & 0.924 \\
RGPF-LGPF & 0.010 & 0.884 \\
IF-GPF & 0.045 & 0.452 \\
MA length & -0.390 & 0.428 \\
MA amplitude & -0.373 & 0.405 \\
Palate size & 0.742 & 0.356 \\
Height & 0.382 & $<0.001$ \\
Superior width & 0.028 & 0.709 \\
Inferior width & 0.117 & 0.238 \\
\hline
\end{tabular}

RGPF-LGPF — distance between the left and right greater palatine foramina; IF-GPF distance between the incisive fossa (IF) and the mid-point between the greater palatine foramina (GPF); MA — maxillary alveolar

Table 6. Mean values (mm), standard deviation (SD) and $p$-value found for the measurements taken of the pyriform aperture, by sex

\begin{tabular}{lccccc}
\hline Pyriform aperture & Sex & N & Mean & SD & P \\
\hline Height & Male & 175 & 33.95 & 3.40 & $<0.001$ \\
& Female & 90 & 30.62 & 3.26 & \\
Superior width & Male & 174 & 16.33 & 2.68 & 0.775 \\
& Female & 90 & 16.23 & 2.45 & \\
Inferior width & Male & 174 & 25.05 & 1.95 & 0.064 \\
& Female & 90 & 24.62 & 2.35 & \\
\hline
\end{tabular}

$\mathrm{N}$ - number of skulls analysed

The coefficient of linear regression showed that only the height is a good predictor of male sex.

Analysis of the model's quality was by percentage of concordant and discordant results; it was assessed as good with $80.6 \%$ concordant results, $19.1 \%$ discordant results and $0.3 \%$ undifferentiated. The model's quality was also analysed by Pearson's correlation ( $p=0.572)$, the Deviance test (0.4119) and the Hosmer-Lemeshow test $(p=0.828)$; all showed that the model was good, with good adherence.

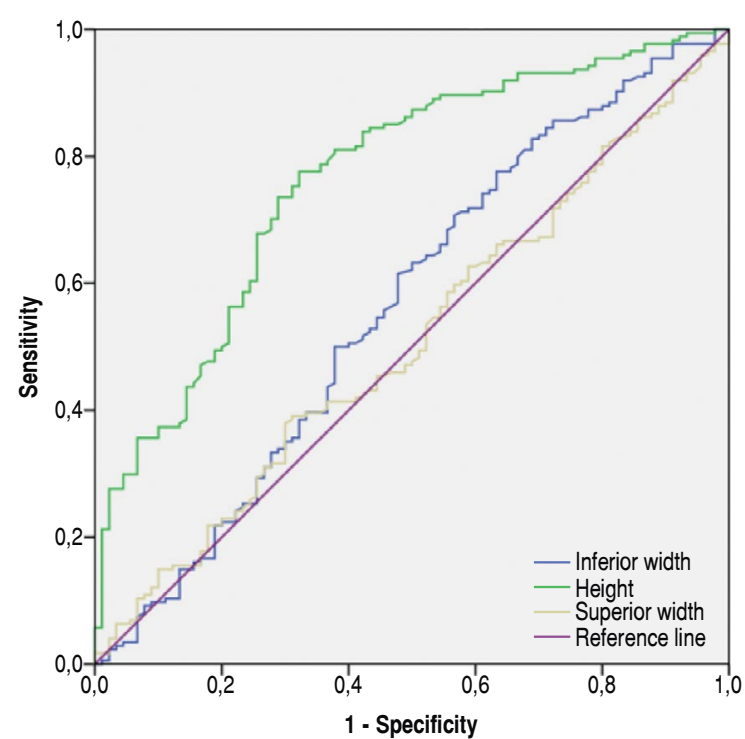

Figure 4. Receiver operating characteristic curve for the measurements taken in the pyriform aperture.

\section{DISCUSSION}

Determining the biological sex of skeletal remains is one of the main tasks of forensic anthropologists for victim identification $[6,11]$. To do this, the forensic anthropologist must combine and compare assessments of different parts of the skeleton $[6,11,27]$, since different regions of the same skeleton may present different levels of sexual expression [5].

The biomechanical load, stress and functions of each part of the body, as well as their hormone levels, can determine morphological differences in the tissues of men and women $[5,20]$. In general, males are larger, with more robust facial characteristics and cranium than females [10]. Previous studies have proposed that the climate can also affect cranium shape, more specifically that the shape of the face changes under extreme climatic conditions [9]. Some authors have related the nutrition and socio-economic status of the individual with osseous morphology and levels of

Table 7. Analysis of the receiver operating characteristic curve for the pyriform aperture

\begin{tabular}{lccccccc}
\hline Pyriform aperture & AUC & CP & Accuracy & Sensitivity & Specificity & PPV & NPV \\
\hline Height & 0.764 & 31,550 & $74.2 \%$ & $67.8 \%$ & $77.6 \%$ & $61.1 \%$ & $82.3 \%$ \\
Superior width & 0.509 & 17,230 & $49.2 \%$ & $39.1 \%$ & $68.9 \%$ & $70.8 \%$ & $36.9 \%$ \\
Inferior width & 0.562 & 23,730 & $63.6 \%$ & $77.6 \%$ & $36.7 \%$ & $70.3 \%$ & $45.8 \%$ \\
\hline
\end{tabular}

AUC — area under curve; $\mathrm{CP}$ — cut-off point; PPV — positive predictive value; NPV — negative predictive value 
sexual dimorphism [3]. By combining two or more of the morphological characteristics of the cranium, high levels of accuracy and precision can be achieved in determining sex [33]. The sex of the individual cannot be conclusively determined without combining more than one dimorphic sexual parameter $[6,11,18,27]$.

In the metric analysis of the pyriform aperture in this study, only the height presented sexual dimorphism, with males presenting higher mean values than females; this finding is corroborated by other authors $[1,4,16]$. The mean values found for the height of the PA in the present study were higher than found in Indians $(31.16 \mathrm{~mm}$ for males and $26.57 \mathrm{~mm}$ for females) [4] and Koreans (30.1 mm for males and $28.0 \mathrm{~mm}$ for females) [16], and lower than reported in Egyptians ( $38.24 \mathrm{~mm}$ for males and $35.12 \mathrm{~mm}$ for females) [1]. The height of the PA was found to be a good predictor for determining sex, corroborating Abdelaleem et al. [1] who reported a good concordance correlation coefficient. Asghar et al. [4] analysed the inferior width of the PA in Indians and reported lower mean values than found in the present investigation $(24.0 \mathrm{~mm}$ for males and $22.77 \mathrm{~mm}$ for females); Saini et al. [26], also in a population of Indians, reported values quite similar to those found in our study $(25.31 \mathrm{~mm}$ for males and $24.97 \mathrm{~mm}$ for females), while Abdelaleem et al. [1] reported higher values in an Egyptian population $(30.53 \mathrm{~mm}$ for males and $27.08 \mathrm{~mm}$ for females) [16]. Moreover, Abdelaleem et al. [1] and Saini et al. [26] reported that the inferior width of the PA offers $67.7-78.6 \%$ accuracy in predicting sex, similar results to those found in the present study where we report $74.2 \%$ accuracy and 0.764 AUC. In the present investigation we found no statistical differences between sexes for the inferior width of the PA, corroborating the findings of Cantín López et al. [7]; however, it should be noted that this measurement presented moderate accuracy, good sensitivity and good PPV for sex diagnosis. Nevertheless, because of its low AUC, specificity and NPV, we do not consider it a good sex predictor. We agree with Hunnargi et al. [15] who state that the conflicting results found for the inferior width of the PA may be due to the fact that precision in sex determination may vary according to the statistical method used. For the superior width of the PA, Cantín López et al. [7] found lower mean values than ours, of $13.29 \mathrm{~mm}$ for males and $12.75 \mathrm{~mm}$ for females; however like us, they found no significant difference between sexes in this dimension. Although the superior width pre- sents good PPV, the present investigation found low accuracy and sensitivity in the sex prediction capacity of this measurement.

In our study, metric analysis of the HP showed that all the measurements present sexual differences, with higher mean values in males than in females, except for RGPF-LGPF. We observed that the RGPF-LGPF was similar to that reported in Indians, with $30.37 \mathrm{~mm}$ for males and 31.01 for females [18]; this absence of sexual dimorphism corroborated earlier studies [18]. The maxillary alveolar amplitudes reported for North Indians were similar to those found in our study for males $(60.36 \mathrm{~mm})$ and inferior to that for females $(56.94$ $\mathrm{mm}$ ) [31]. In this study the maxillary alveolar amplitude presented the greatest balance between sensitivity and specificity, with good PPV and moderate accuracy. We found that the maxillary alveolar length was substantially superior to that reported for North Indians, with $51.55 \mathrm{~mm}$ for males and $48.81 \mathrm{~mm}$ for females [31], with a significant difference between the sexes. The values reported for palate size in North Indians (31.15 mm for males and $27.86 \mathrm{~mm}$ for females) [31] were substantially lower than those found in our study. Sumati et al. [31] reported that palate size is excellent for determining sex; our findings disagreed, since although this measurement presents sexual dimorphism and good specificity, the values for sensitivity and accuracy were low. IF-GPF was the measurement which showed the greatest accuracy and sensitivity of all the measurements analysed in this study, but with low specificity. This finding is important for forensic scientists because in some situations the alveolar margins may be pathologically deformed or fragmented by a trauma which makes cranial sexing by this anatomical structure unusable; whereas the median region of the palate, from the incisive fossa to the posterior nasal spine, is very resistant to trauma and high temperatures [18]. Although palate size is not an excellent indicator of sex, it can be used as an auxiliary resource for sexing in extreme situations when only fragments of bone are available for analysis.

The PA and the HP are important for sexual differentiation in cases when only bone fragments of the individual are accessible, since these two characteristics play a significant part in sex diagnosis [33]. In morphological analysis of the PA and the HP, we observed that the PA presents greater accuracy and better balance between sensitivity and specificity, making it a better sex predictor than the HP. The 
binary logistic regression showed that the model using the HP was not significant, while the model using the PA was significant but weak. Williams and Rogers [33] found greater accuracy for the shape of the PA, with $84 \%$. In a ranking of 1-9 for combined precision plus accuracy, Williams and Rogers [33] classified the PA as the third best morphological characteristic of the cranium for sex prediction and the HP as the seventh best. We agree with Rogers [25] and Williams and Rogers [33] when they say that palate shape did not perform well for accuracy of sexual identification.

In the present investigation we carried out metric and non-metric analysis of the PA and HP in a sample of macerated skulls with information on sex. By using populations of known sex, the researcher has the advantage of being able to evaluate techniques and determine their precision and applicability [33]. It should be noted that, in our investigation, metric analysis proved a more reliable predictor of sex than non-metric analysis. The non-metric methods used to determine sex depended on visual assessment of sexually dimorphic features [23]; although these features present rapid preliminary results, they are influenced by the observer's subjectivity [19], and must be carried out by an experienced observer for precise analysis [21]. Metric methods are based on the basic principle of variations in physical dimensions between males and females. Different statistical methods are used to derive models/equations which can be used for sexing $[17,24]$. In this study, sex prediction by analysis of the morphological characteristics of the PA was better than by measuring the HP, with greater accuracy and sensitivity/specificity ratio. Metric analysis showed that both structures present sexual dimorphism; however, the height of the pyriform aperture was the measurement which showed the greatest accuracy and the best sensitivity/specificity ratio for diagnosing sex, and was the best sex predictor of the measurements evaluated in this study.

\section{CONCLUSIONS}

Non-metric analysis of the PA presented better accuracy and sensitivity/specificity ratio than HP, and can be used in association with other parameters as an additional tool for sex determination, especially when only bone fragments are available for sexing. Metric analysis of the HP showed sexual dimorphism in this structure; however, the height of the PA was the most reliable indicator for sex prediction, and could be used by forensic scientists to identify sex.

\section{Acknowledgements}

Thanks for Universidad de La Frontera.

\section{REFERENCES}

1. Abdelaleem $S$, Younis $R$, Kader $M$. Sex determination from the piriform aperture using multi slice computed tomography: Discriminant function analysis of Egyptian population in Minia Governorate. Egypt J Forensic Sci. 2016; 6(4): 429-434, doi: 10.1016/j.ejfs.2016.11.003.

2. Alves N, Cândido P. Anatomia para o curso de odontologia geral e específica, 4th edition. São Paulo: Editora Santos. 2016.

3. Angel JL. Colonial modern skeletal change in the U.S.A. Am J Phys Anthropol. 1976; 45(3 Pt. 2): 723-735, doi: 10.1002/ ajpa.1330450339, indexed in Pubmed: 793421.

4. Asghar A, Dixit A, Rani M. Morphometric study of nasal bone and piriform aperture in human dry skull of indian origin. J Clin Diagn Res. 2016; 10(1): AC05-AC07, doi: $10.7860 / J C D R / 2016 / 15677.7148$, indexed in Pubmed: 26894050.

5. Best KC, Garvin HM, Cabo LL. An Investigation into the Relationship between Human Cranial and Pelvic Sexual Dimorphism. J Forensic Sci. 2018; 63(4): 990-1000, doi: 10.1111/1556-4029.13669, indexed in Pubmed: 29044526.

6. Cabo L, Brewster C, Azpiazu JL. Sexual dimorphism: interpreting sex markers. A Companion to Forensic Anthropology. Chichester, UK: John Wiley \& Sons, Ltd. 2012: 248-286, doi: 10.1002/9781118255377.ch13.

7. Cantín López M, Galdames IS, Matamala DZ, et al. Sexual dimorphism determination by piriform aperture morphometric analysis in brazilian human skulls. Int J Morphol. 2009; 27(2): 327-331, doi: 10.4067/s071795022009000200007.

8. Cattaneo C. Forensic anthropology: developments of a classical discipline in the new millennium. Forensic Sci Int. 2007; 165(2-3): 185-193, doi: 10.1016/j.forsciint.2006.05.018, indexed in Pubmed: 16843626.

9. Evteev A, Cardini AL, Morozova I, et al. Extreme climate, rather than population history, explains mid-facial morphology of Northern Asians. Am J Phys Anthropol. 2014; 153(3): 449-462, doi: 10.1002/ajpa.22444, indexed in Pubmed: 24374801.

10. Frayer DW, Wolpoff MH. Sexual dimorphism. Annu Rev Anthropol. 1985; 14(1): 429-473, doi: 10.1146/annurev. an.14.100185.002241.

11. Garvin H. Adult sex determination: methods and application. A Companion to Forensic Anthropology. 2012: 239-247, doi: 10.1002/9781118255377.ch12.

12. Gonçalves D, Thompson TJU, Cunha E. Osteometric sex determination of burned human skeletal remains. J Forensic Leg Med. 2013; 20(7): 906-911, doi: 10.1016/j. jflm.2013.07.003, indexed in Pubmed: 24112343.

13. Holland TD. Use of the cranial base in the identification of fire victims. J Forensic Sci. 1989; 34(2): 458-460, indexed in Pubmed: 2708959. 
14. Hommerich $C P$, Riegel $A$. Measuring of the piriform aperture in humans with 3D-SSD-CT-reconstructions. Ann Anat. 2002; 184(5): 455-459, doi: 10.1016/509409602(02)80078-4, indexed in Pubmed: 12392325.

15. Hunnargi SA, Menezes RG, Kanchan T, et al. Sternal index: Is it a reliable indicator of sex in the Maharashtrian population of India? J Forensic Leg Med. 2009; 16(2): 56-58, doi: 10.1016/j.jflm.2008.08.004, indexed in Pubmed: 19134997.

16. Hwang TS, Song J, Yoon Ho, et al. Morphometry of nasal bases and nostrils in Koreans. Ann Anat. 2003; 185(2): 189-193, doi: 10.1016/S0940-9602(03)80088-2, indexed in Pubmed: 12725444.

17. Yaşar Işcan M, Steyn M. Craniometric determination of population affinity in South Africans. Int J Legal Med. 1999; 112(2): 91-97, indexed in Pubmed: 10048665.

18. Kamath V, Asif M, Shetty R, et al. Binary logistic regression analysis of hard palate dimensions for sexing human crania. Anat Cell Biol. 2016; 49(2): 151-159, doi: 10.5115/ acb.2016.49.2.151, indexed in Pubmed: 27382518.

19. Kemkes-Grottenthaler A, Löbig F, Stock F. Mandibular ramus flexure and gonial eversion as morphologic indicators of sex. Homo. 2002; 53(2): 97-111, indexed in Pubmed: 12489410.

20. Klepinger LL. Stature, maturation variation and secular trends in forensic anthropology. J Forensic Sci. 2001; 46(4): 788-790, indexed in Pubmed: 11451057.

21. Krishan K, Chatterjee PM, Kanchan T, et al. A review of sex estimation techniques during examination of skeletal remains in forensic anthropology casework. Forensic Sci Int. 2016; 261: 165.e1-165.e8, doi: 10.1016/j.forsciint.2016.02.007, indexed in Pubmed: 26926105.

22. Larnach S, Macintosh N. The craniology of the Aborigines of Queensland, Sydney: Australasian Medical Pub. Co. for the University of Sydney. 1970.

23. Mahfouz M, Badawi A, Merkl B, et al. Patella sex determination by $3 \mathrm{D}$ statistical shape models and nonlinear classifiers. Forensic Sci Int. 2007; 173(2-3): 161-170, doi: 10.1016/j. forsciint.2007.02.024, indexed in Pubmed: 17482786.
24. Pretorius E, Steyn M, Scholtz Y. Investigation into the usability of geometric morphometric analysis in assessment of sexual dimorphism. Am J Phys Anthropol. 2006; 129(1): 64-70, doi: 10.1002/ajpa.20251, indexed in Pubmed: 16245344.

25. Rogers TL. Determining the sex of human remains through cranial morphology. J Forensic Sci. 2005; 50(3): 493-500, indexed in Pubmed: 15932077.

26. Saini V, Srivastava R, Rai RK, et al. An osteometric study of northern Indian populations for sexual dimorphism in craniofacial region. J Forensic Sci. 2011; 56(3): 700-705, doi: 10.1111/j.1556-4029.2011.01707.x, indexed in Pubmed: 21361935.

27. Spradley MK, Jantz RL. Sex estimation in forensic anthropology: skull versus postcranial elements. J Forensic Sci. 2011; 56(2): 289-296, doi: 10.1111/j.15564029.2010.01635.x, indexed in Pubmed: 21210801.

28. Standring S. Gray's anatomy: the anatomical basis of clinical practice, 40th edition. Churchill Livingstone. 2008.

29. Steadman DW, Haglund WD. The scope of anthropological contributions to human rights investigations. J Forensic Sci. 2005; 50(1): 23-30, indexed in Pubmed: 15830993.

30. Stewart T. Essentials of forensic anthropology. IL C C Thomas, Springfield 1979.

31. Sumati, Patnaik V, Phatak A. Determination of sex from hard palate by discriminant. Int J Basic Appl Med Sci. 2012; 2(3): 243-251.

32. Wilkson C. Forensic Facial Reconstruction. University Press, Cambridge 2004: 292.

33. Williams BA, Rogers T. Evaluating the accuracy and precision of cranial morphological traits for sex determination. J Forensic Sci. 2006; 51(4): 729-735, doi: 10.1111/j.15564029.2006.00177.x, indexed in Pubmed: 16882212.

34. Wolpoff $M H$. Climatic influence on the skeletal nasal aperture. Am J Phys Anthropol. 1968; 29(3): 405-423, indexed in Pubmed: 5710378. 